Purdue University

Purdue e-Pubs

\title{
Piezoelectric Fans using Higher Flexural Modes for Electronics Cooling Applications
}

Sydney M. Wait

Sudipta Basak

S V. Garimella

Purdue University, sureshg@purdue.edu

Arvind Raman

Follow this and additional works at: http://docs.lib.purdue.edu/coolingpubs

Wait, Sydney M.; Basak, Sudipta; Garimella, S V.; and Raman, Arvind, "Piezoelectric Fans using Higher Flexural Modes for Electronics Cooling Applications" (2007). CTRC Research Publications. Paper 53.

http://dx.doi.org/10.1109/TCAPT.2007.892084

This document has been made available through Purdue e-Pubs, a service of the Purdue University Libraries. Please contact epubs@purdue.edu for additional information. 


\title{
Piezoelectric fans using higher flexural modes for electronics cooling applications
}

\author{
Sydney M. Wait, Sudipta Basak, Suresh V. Garimella, and Arvind Raman
}

\begin{abstract}
Piezoelectric fans are gaining in popularity as lowpower-consumption and low-noise devices for the removal of heat in confined spaces. The performance of piezoelectric fans has been studied by several authors, although primarily at the fundamental resonance mode. In this article the performance of piezoelectric fans operating at the higher resonance modes is studied in detail. Experiments are performed on a number of commercially available piezoelectric fans of varying length. Both finite element modeling and experimental impedance measurements are used to demonstrate that the electromechanical energy conversion (electromechanical coupling factors) in certain modes can be greater than in the first bending mode; however, losses in the piezoceramic are also shown to be higher at those modes. The overall power consumption of the fans is also found to increase with increasing mode number. Detailed flow visualizations are also performed to understand both the transient and steady-state fluid motion around these fans. The results indicate that certain advantages of piezoelectric fan operation at higher resonance modes are offset by increased power consumption and decreased fluid flow.
\end{abstract}

Index Terms-acoustic devices, fans, air cooling, electromechanical effects, losses, piezoelectric devices.

\section{INTRODUCTION}

$\mathrm{P}$ ORTABLE electronic devices have steadily gained in popularity over the last two decades. Cell phones, portable digital assistants (PDAs), and laptops are considered essential in much of the world today for business communications and information processing. With increased popularity has come an increased demand for greater functionality, larger storage space, faster rates of information transfer and the availability of more features in a single device. Advances in wireless technology have also fueled the need for invention and innovation in the field of portable electronics.

As more electronics are packed into smaller spaces, the heat removal requirements for these devices become increasingly demanding. Not only is there more heat to remove, but size constraints and limits on power consumption of the cooling mechanism have framed the need for more efficient cooling solutions.

Manuscript received May 16, 2005. This work was supported by the Cooling Technologies Research Center at Purdue University, the National Science Foundation, and the Semiconductor Research Corporation

All the authors are with the School of Mechanical Engineering at Purdue University, West Lafayette, Indiana, USA.

Address correspondence to S. V. Garimella (sureshg@ purdue.edu).
Cooling techniques that can meet the special needs of the portable electronics industry are essential to continued progress in this field. Piezoelectric fans are one innovative technology that are gaining acceptance as a viable solution $[1,2]$. These fans generally consist of a patch of piezoelectric material attached to one or both sides of a shim stock as illustrated in Fig. 1. When an alternating voltage is applied to the patch, it expands and contracts in the lengthwise direction at the frequency of the input. This effectively applies bending moments at the beginning and end of the patch. These moments cause the beam to oscillate flexurally at the same frequency as the applied voltage. The amplitude of oscillation is maximized if the input voltage is applied to the patch at the resonance frequency of the fan. This oscillatory motion generates air flow that can be exploited for cooling.

The mechanism by which an oscillating beam generates air flow has been studied by several authors. Toda [3] predicted the resonance frequency and flow rate for a $\mathrm{PVF}_{2}$ (poly vinylidene diflouride) piezoelectric fan and verified his calculations against experiments. The experimental and analytical results were in good agreement, particularly for larger fans. Ihara and Watanabe [4] investigated the twodimensional flow around the ends of flexible plates oscillating with large amplitudes. They primarily focused on the unsteady flow and vortex generation from these fans, and did not explicitly discuss the steady streaming flows generated. Nyborg [5] developed a numerical approximation for acoustic streaming near an oscillating boundary which is valid when the boundary layer parameter $\sqrt{v / \omega}$ (where $v$ is the kinematic viscosity and $\omega$ the angular frequency) is much smaller than the amplitude scale of the oscillation velocity. Kim et al. [6] used PIV techniques and computational methods to quantitatively verify the velocities and vortex generation from the end of a vibrating cantilever. They found that instantaneous velocities in the flow field were up to four times greater than the tip velocity, and that three-dimensional effects from the vortex shedding were significant. Acikalin et al. [7] investigated two-dimensional streaming flows around oscillating thin beams using analytical, computational, and experimental methods. The predicted flow patterns from a baffled piezoelectric fan vibrating in its first mode were verified using flow visualization. Acoustic streaming in flexural plate wave (FPW) devices was investigated by Nguyen and White [8]. They developed two- and threedimensional numerical models of acoustic streaming and 
investigated the influence of wave amplitude and back pressure on the time-averaged velocity in the flow field.

The flow generated by vibrating beams has been studied by several authors as a mechanism by which to enhance heat transfer [1,2, 9-13]. Significant promise has been shown for utilizing piezoelectric fans operating at their first resonance mode for cooling. However the cooling capabilities of fans operating at higher resonance modes have not been widely studied. More complex mode shapes may enhance mixing, thereby increasing convection near a heat source. It is also possible that higher frequencies associated with higher resonance modes could increase the streaming flow velocity of the fan. Higher resonance modes may also display a more effective conversion of electrical energy to mechanical energy. However, it remains unclear how the resonance mode affects the power consumption and losses in the fan system. In order for fan operation at a higher resonance mode to be exploited in cooling applications, any benefits to heat transfer gained by operating the fan at that mode should not be offset by an increase in the power consumption of the fan.

In what follows, the above issues are addressed through detailed experimental investigations and finite element modeling. Flow visualizations are performed with three commercial fans, the materials and dimensions of which are listed in Table I. The effect of resonance mode on the electromechanical energy conversion, the power consumption and self-heating of the fans, as well as on the flow generated by the fans, is studied in detail.

\section{ELECTROMECHANICAL COUPLING FACTORS}

A piezoelectric fan is a composite electromechanical structure that converts electrical potential into mechanical strain. In order to understand the effectiveness of the conversion of electrical to mechanical energy, consider a simple equivalent electrical circuit model of the piezoelectric fan shown in Fig. 2 (see for example [14]). The resistance in the equivalent circuit represents the irreversible leakage of electrical energy into electrical and mechanical dissipation, and the inductance represents the mass of the oscillator. The capacitance, $\mathrm{C}_{\mathrm{o}}$, corresponds to the capacitance of the piezoceramic patch and represents the electrical energy stored therein, whereas the capacitance $\mathrm{C}$ represents the storage of strain energy in the structure.

Such a circuit possesses two "natural" frequencies, namely $\mathrm{f}_{n}$ and $\mathrm{f}_{m}$, corresponding to the maximum and minimum of the absolute value of the circuit impedance. The percentage of electrical energy that can be converted to mechanical energy in such an electromechanical system can be shown to be directly proportional to the dynamic electromechanical coupling factor (EMCF) of the circuit [15]:

$E M C F=\left(\frac{f_{n}^{2}-f_{m}^{2}}{f_{n}^{2}}\right)^{1 / 2}$,

In (1), $\mathrm{f}_{m}$ represents the short-circuited series resonance frequency of the $R-L-C$ branch in Fig. 2, whereas $\mathrm{f}_{n}$ represents the open-circuit resonance frequency of the complete circuit. This simple electrical analogy is truly valid for an electromechanical oscillator with a single resonance mode. However, a piezoelectric fan is a continuous system with an infinite number of natural frequencies and mode shapes. In principle, each resonance mode of the piezoelectric fan will have its own specific equivalent circuit, and EMCF value.

The accurate prediction and measurement of the short- and open-circuited resonance frequencies and mode shapes of a piezoelectric fan are important from several points of view. Firstly, when the piezoelectric fan is driven at a short-circuited resonance frequency, the equivalent circuit impedance is minimized locally, resulting in greater mechanical energy or larger amplitudes of fan vibration. For this reason, piezoelectric fans should always be driven near their shortcircuited resonance frequencies. Secondly, the difference between the open- and short-circuited resonance frequencies provides a measure of the conversion effectiveness of the input electrical energy into mechanical oscillation of the fan [15-17]. Lastly, the prediction of mode shapes themselves is important because it is directly correlated to the flow field surrounding the fan.

The resonance frequencies of the fan can be determined by several methods, three of which were used in this work: (a) using finite element analysis to predict the resonance frequency based on electromechanical characteristics; (b) measuring the impedance of the piezoelectric fan circuit as a function of frequency; and (c) qualitative observation of the flow produced by the fan to determine the frequency at which maximum flow is achieved.

The finite element model considers a piezoelectric fan divided into three regions as shown in Fig. 3. The piezoceramic patch has a total length L2 and extends a distance $\mathrm{L} 1$ beyond the left end of the mylar shim; the mylar shim extends beyond the piezopatch from L2 to L3. Finite element models were created for the three different fans studied experimentally, the geometies of which are specified in Table I. The finite element computations were performed in ANSYS 8.0 [18] using SOLID5 elements. SOLID5 is a 20node three-dimensional linear coupled-field solid element having both piezoelectric and structural properties. The model is constructed by first defining keypoints, creating volumes, and assigning the appropriate material properties to the respective volumes. The constitutive properties used for the computation are those of mylar [19] (Young's modulus of 4.6 $\mathrm{GPa}$, Poisson's ratio of 0.44 and density of $1240 \mathrm{~kg} / \mathrm{m}^{3}$ ) and Navy Type II PZT [20] The electromechanical properties of the piezoceramic are presented in matrix form below: 


$$
\begin{aligned}
c & =\left[\begin{array}{cccccc}
6.6 & 3.3 & 3.3 & 0 & 0 & 0 \\
3.3 & 6.6 & 3.3 & 0 & 0 & 0 \\
3.3 & 3.3 & 5.2 & 0 & 0 & 0 \\
0 & 0 & 0 & 1 & 0 & 0 \\
0 & 0 & 0 & 0 & 1 & 0 \\
0 & 0 & 0 & 0 & 0 & 1
\end{array}\right] 10^{10} P a \\
\varepsilon & =\left[\begin{array}{ccc}
1800 & 0 & 0 \\
0 & 1800 & 0 \\
0 & 0 & 1800 \\
0 & 0 & 0 \\
0 & 0 & 0 \\
0 & 0 & 0
\end{array}\right] \varepsilon_{0} F / m \\
& =\left[\begin{array}{ccc}
-5.58 & 0 & 0 \\
0 & -5.58 & 0 \\
0 & 0 & 7.72
\end{array}\right] C / m^{2}
\end{aligned}
$$

in which $\boldsymbol{c}, \boldsymbol{\varepsilon}, \boldsymbol{e}$ are the mechanical modulus, permittivity, and piezoelectric matrices, and $\varepsilon_{0}$ is the permittivity of free space.

A perfect, infinitesimal-thickness bond is assumed between the piezoceramic patch and the mylar beam and the model is also assumed to be loss-less. This loss-less assumption will be shown later to introduce a small, but acceptable source of error while comparing theoretical and experimental results. A free three-dimensional mesh with maximum edge length of $0.8 \mathrm{~mm}$ is constructed which yields a regular brick mesh (Fig. 3). Further analysis indicated that this mesh density is sufficiently dense for accurate predictions of the frequencies and mode shapes of the first four flexural modes of the fan. All nodes underneath and above the piezopatch are assigned a voltage coupling for the short-circuited (SC) and open-circuited (OC) free vibration problem (modal analysis). For the SC condition, the top electrode is assigned zero voltage, while for the OC case, the voltages at all nodes on the top electrode are forced to be identical. The frequency predictions of the finite element model are presented in Table II, and the modal deformations of the fans at each mode are shown in Fig. 4.

In addition to the FE calculations for the three fans, an impedance analyzer (HP 4294) is used to determine their resonance frequencies experimentally. Local maxima and minima in the plot of impedance vs. frequency are assumed to represent open- and short-circuit frequencies, respectively; the first local minimum in the impedance curve corresponds to the first resonance mode of the fan and subsequent dips correspond to higher resonance modes. The short-circuited resonance frequencies are also determined by tuning the frequency of the fan and finding the point at which the flow generated appears to be the maximum. The predicted, measured (impedance analyzer) and observed (flow visualization) resonance frequencies for Fans 1, 2, and 3 are compared in Table II. Except for the first mode of Fan 3, the measured values agree with the ANSYS predictions to within $5 \%$ for all the fan modes, and are very close to the frequencies at which maximum flow occurs. The third mode of Fan 2 was predicted to occur at a frequency of $600 \mathrm{~Hz}$. However, this is a mode that did not generate sufficient flow for observation, which might be due to the very low electromechanical energy conversion at this mode.

The dynamic EMCF of a loss-less resonator is given by

$E M C F=\left(\frac{f_{p}^{2}-f_{s}^{2}}{f_{p}^{2}}\right)^{1 / 2}$,

where $f_{p}$ and $f_{s}$, called the parallel and series resonance frequencies respectively, are the frequencies corresponding to the maximum and minimum of the circuit impedance for the loss-less resonator. This is in contrast to (1) which accounts for losses in the piezoceramic. The experimentally measured EMCFs calculated using (1), and the FE-predicted EMCFs calculated using (2) are listed in Table II.

In general, the finite element predicted EMCFs underpredict those measured experimentally. This is an expected result and can be explained as follows. For a loss-less resonator the OC and $\mathrm{SC}$ frequencies are equivalent to the parallel $\left(\mathrm{f}_{p}\right)$ and series $\left(\mathrm{f}_{s}\right)$ resonance frequencies of the simple piezoelectric equivalent circuit in Fig. 2 [15,17]. With the introduction of losses (represented by $\mathrm{R}$ in the figure) this equivalence no longer holds and two pairs of characteristic frequencies $\left(f_{p}, f_{s}\right.$, $\left.f_{m}, f_{n}\right)$ appear. The difference between the frequencies at maximum and minimum impedance $\left(f_{n}-f_{m}\right)$ can be related to the frequency difference $\left(f_{p}-f_{s}\right)$ by Eq. 138 from IRE Standards on Piezoelectric Crystals [17].

$$
\Delta f=f_{p}-f_{s}=\frac{f_{n}-f_{m}}{\left(1+\frac{4}{M^{2}}\right)^{1 / 2}}
$$

where $\mathrm{M}$, the figure of merit is

$$
M=\frac{1}{2 \pi f_{s} R C_{o}} \text {. }
$$

Thus as the losses increase (or $\mathrm{R}$ increases), $\mathrm{M}$ decreases and the frequency difference $\left(f_{p}-f_{s}\right)$ becomes lesser than $\left(f_{n}-f_{m}\right)$. This results in an overprediction of EMCF when it is calculated from the experimentally measured frequencies corresponding to the maximum and minimum of the impedance plot for the fan with losses. The ANSYS model consistently predicts a lower value of EMCF compared to the experiment because of the unaccounted losses in the fan structure in the finite element model. This means that the EMCF calculated from experiments and that predicted using the frequencies obtained from the finite element model serve as the upper and lower bounds, respectively, for the true value of EMCF. This fact was also observed in the experiments of [16].

The EMCF values for the three fans based on experiments and finite element predictions (Table II) reveal that the EMCF is maximized at mode 2 for Fan 1 and Fan 2. This interesting result can be explained by computing in the FE model the ratio 
of the strain energy in the piezopatch to that of the total strain energy in Fan 1 (Fig. 5). The variation of the EMCF with mode number follows the same pattern as this energy ratio. For the particular configuration of the fan considered in this work, this energy ratio is maximized for the second mode of vibration. As the energy available in the piezopatch increases, the convertible energy of the fan and thereby EMCF would be expected to increase. The results presented thus far suggest that operation in the second bending mode may be favorable from the point of view of effectiveness of conversion of electrical to mechanical energy. In what follows, some important disadvantages to higher-mode operation are discussed.

\section{POWER CONSUMPTION AND LOSSES}

In portable electronics, which is the primary target market for using piezoelectric fans for cooling, power consumption is a critical issue that can enhance or nullify any advantages to operating at higher resonance modes. In order to more fully characterize the performance of piezoelectric fans at higher resonance modes, the dependence of power consumption and losses in the fan circuit on choice of the operating resonance mode must be understood.

The power consumption is calculated using the following equation [21]:

$P=1 / t \int_{0}^{t} v\left(t^{\prime}\right) i\left(t^{\prime}\right) d t^{\prime}$,

where $\mathrm{t}$ is the time period over which the signal is measured. The voltage and current input to the piezoelectric fan are determined by placing a $1 \mathrm{k} \Omega$ resistor in series between the piezoelectric fan and ground, and measuring the voltage drop between the power supply and ground, and across the resistor, as shown in Fig. 6. The time-varying voltage input, $v(t)$, is measured as $\Delta \mathrm{V}_{1}$ and the time-varying current, $i(t)$, is measured as $\Delta \mathrm{V}_{2} / \mathrm{R}$ using a digital oscilloscope. The resulting waveforms are integrated numerically to obtain the power consumed by the fan. Measurements of power consumption for Fans 1, 2, and 3 operating at the first several resonance modes are summarized in Table II. The power consumption is more than eight times greater at the second mode than the first mode for Fans 1 and 2, and it continues to increase with mode number.

Piezoelectric fans exhibit mechanical, dielectric and piezoelectric losses [22]. These losses are best understood through the equivalent electrical circuit [14] with resistance $(\mathrm{R})$, inductance $(\mathrm{L})$, and parallel capacitance $\left(\mathrm{C}, \mathrm{C}_{\mathrm{o}}\right)$ values representing the dissipation, inertial, and storage terms for the system. The resistance contributes to the resistive or the real part of the equivalent circuit impedance, while the inductance and capacitance contribute to the reactive or imaginary part. The series impedance, $Z_{s}$, through the series branch is given by:

$Z_{s}=R+j \omega L+1 / j \omega C$, and the total or parallel impedance, $Z_{p}$, is given by

$Z_{p}=\frac{\left(Z_{s}\right)\left(1 / j \omega C_{o}\right)}{Z_{s}+1 / j \omega C_{o}}$,

Fig. 7 shows a schematic representation of the impedance in the real-imaginary plane. A loss-less resonator would be purely reactive and all the power supplied to the circuit will be returned to the source. The total loss in the system is quantified as the tangent of the angle between the imaginary axis and the magnitude of the impedance, i.e., $\tan \alpha$. It is noted that $\tan \alpha$ is zero when the impedance is purely reactive, and the larger the angle $\alpha$, the greater is the loss in the system.

The experimentally measured loss factor tan $\alpha$ vs. driving frequency for the first resonance mode of Fan 2 is shown in Fig. 8. The experimentally measured impedance is also plotted on the same graph. Clearly the losses as quantified by $\tan \alpha$ are maximized when the driving frequency is between the OC and SC resonance frequencies of the mode. Similar plots have been generated at each resonance mode for all three fans used in this study and it is found consistently that the loss factor is largest when operating between the OC and SC frequency. This is a significant observation because it demonstrates that operating slightly above the SC resonance may dramatically increase the losses in the fan system. Operation at or near resonance is critical for maximizing displacement amplitude, thereby creating the maximum amount of flow. However care must be taken to ensure that the fan is excited at or slightly below the SC resonance frequency to minimize the losses while still providing the maximum amplitude for generating flow.

The loss factors $(\tan \alpha)$ are summarized in Table II for Fans 1 and 2 at the first four resonance modes, and the first mode for Fan 3. For Fans 1 and 2, the losses increase with mode number, though this increase is not monotonic. Because the equivalent circuit is unique for each mode, a fluctuating pattern in the loss factor is not unexpected. Although the EMCF is greatest at the second mode for Fans 1 and 2, the larger loss at this mode combined with an increase in power consumption indicates that operation at the second and higher modes may not be preferable to operation at the first mode for the application under study. Observation of the fluid flow generated at each mode (as discussed below) will provide a more sound basis for determining if this is the case.

\section{Flow VisualizATION}

Flow visualization is performed to identify the effects of resonance mode on the flow patterns generated by the piezoelectric fans. The fans are excited with $40 \mathrm{~V}_{\mathrm{rms}}$ at each of the first few resonance frequencies and the resulting flow patterns are observed and recorded. The power consumption is measured at each resonance mode, and the loss factors and EMCF are calculated from impedance data. Results from this section are also included in Table II. 


\section{A. Experimental Setup}

The setup used for each of the flow visualization experiments consists of a piezoelectric fan sandwiched between a transparent plexiglass sheet and a black matte background. The distance between the plexiglass and the matte board is approximately the same as the width of the fan to minimize three-dimensional effects from the fan motion. The size of the domain is $80 \mathrm{~cm}$ by $90 \mathrm{~cm}$, and the boundaries are far enough from the fan to have little effect on the flow field. The flow is seeded with smoke from a theatrical fog generator, and illuminated with light from an Argon-ion laser coupled with sheet fiber optics. The laser sheet illuminates the field from one side, in a plane parallel to the top plexiglass surface, and a digital video camera is positioned above the setup to view the flow field generated by the fan.

An excitation voltage of $40 \mathrm{~V}_{\mathrm{rms}}$ is used for all three fans at the resonance modes given in the "Observed" column of Table II. The flow field is captured with the digital video camera, and still frames are extracted at known times to show the progression of the fluid from the time the fan is turned on, to time $t=1 \mathrm{~s}$. The ANSYS predicted mode shape of each fan is shown in the top left corner for each series of pictures.

\section{B. Flow generated by Fan 1:}

Fig. 9 shows the transient flow produced by Fan 1 operating at its first resonance mode. Vortices are created just before the tip of the fan and are pushed away from the fan as the blade cycles through multiple oscillations. Air is pulled from the right to left in the figure to compensate for the vortices being shed at the tip. This produces a combination of unsteady flow near the tip of the fan, and steady streaming flow across the fan blade.

Fig. 10 shows Fan 1 operating at its second resonance mode. In this figure, the vortices are generated near the midpoint of the fan, corresponding to an antinode of vibration. The vortices are pushed upward and downward away from the fan as it cycles through further oscillations and air is drawn from the right to compensate for the flow being expelled perpendicular to the fan blade. The third resonance mode of Fan 1 is shown in Fig. 11. Similar to the behavior at the second resonance mode shown in Fig. 10, fluid is ejected perpendicular to the fan blade, but the location of the fluid ejection has shifted closer to the tip of the beam for this third resonance mode. This is because the antinode for mode 3 is closer to the free end of the beam than for mode 2 as seen in Fig. 4 (a). Fig. 12 shows Fan 1 operating at its fourth resonance mode. The flow generated is much more localized and steady in this case. Three distinct areas of recirculation are observed, which correspond to the three antinodes along the fan blade seen in Fig. 4 (a); the last recirculation region occurs at the tip of the fan.

As observed earlier, the EMCF for Fan 1 is highest at the second mode; observation of the flow produced at the second mode (Fig. 10) shows that the quality of flow produced at this mode is comparable to the first mode (Fig. 9). However the loss factor is also higher at the second mode than both the first or third mode, and the power consumption is more than eight times greater at the second mode than at the first. While the flow generation decreases from mode 2 onward, the power consumption continues to increase with mode number. This is because the impedance of the fan circuit decreases as the frequency increases, allowing more current to be drawn for the same voltage input. It appears that the first mode of operation for Fan 1 would be the most efficient option when power consumption and losses are also taken into consideration.

\section{Flow generated by Fan 2:}

Fig. 13 shows Fan 2 operating at its first resonance mode. The results are very similar to Fan 1 operating at its first mode. Vortices are generated just before the tip and fluid is pulled from the right side of the figure as the vortices are shed and pushed away from the fan. Fig. 14 shows the flow generated by Fan 2 operating at its second resonance mode. Vortices are generated both near the midpoint and the tip of the beam corresponding to the antinodes of the fan. However, the vortices are not shed, unlike the observation with Fan 1. Rather, they grow into large areas of quasi-steady recirculation at the tip and on either side of the beam. Fig. 15 shows the flow field for Fan 2 operating at its fourth resonance mode. The flow is steady, and there are distinct areas of recirculation corresponding to the antinodes of the beam.

As seen with Fan 1, the EMCF is greatest at the second mode for Fan 2. However the loss factor is also greater at the second mode than the first or third mode and the power consumption is more than six times greater than at the first mode of Fan 2. The power consumption again increases with mode number as the fluid flow decreases from mode 2 onward. No flow was observed at the third resonance mode for Fan 2, most likely because the EMCF is very small at that mode. As with Fan 1, first-mode operation of Fan 2 is most efficient.

\section{Flow generated by Fan 3:}

Fig. 16 shows the flow field generated by Fan 3 operating at its first resonance mode. The flow is steady and distinct areas of recirculation are formed on either side of the beam. The length of the flexible shim is short compared to the length of the piezopatch, which results in a small tip deflection. Because the tip deflection is small, the flow remains fairly steady and the effect of the fan on the fluid is localized. No flow was observed at higher resonance modes for Fan 3, most likely because of the very low EMCF at those modes. The flow produced by Fan 3 is significantly less than that produced by Fans 1 or 2, which indicates that shorter fan lengths have a reduced ability to generate flow.

\section{E. General flow field results}

The flow patterns generated at different resonance modes of the three fans change according to the deformation modeshape of the fan. Areas of recirculation are created between two nodes of the fan and vortices are shed from the tip of the fan. The positions of the vortices correlate well with the ANSYSpredicted deformation of the fans at each resonance mode and 
follow what one would expect given the shape of the deformation.

The bulk fluid motion tends to decrease as the mode number increases primarily because the amplitude of fan vibration decreases. Additionally, as the motion of the fan becomes more complex along the length of the blade, different portions of the fan blade moving in opposing directions tend to counteract, rather than augment, flow in a given direction. Furthermore, decreasing the length of the fan also tends to decrease the flow volume because the amplitude of vibration is smaller, and the surface area available to "push" the fluid decreases.

\section{CONCLUSIONS}

It may be expected that the operation of piezoelectric fans at higher resonance modes would lead to greater streaming flows that are spatially complex, thereby leading to greater fluid mixing and cooling. In particular, fluid motion is shown in this work to be displaced at beam antinodes and sufficient amplitudes result in unsteady streaming from these antinodes. Moreover, the EMCF of a piezoelectric fan can be greater at higher resonance modes (in this case it was maximum at the second resonance mode for Fans 1 and 2). In practice, however, detailed experimental investigations indicate that these advantages are offset by serious disadvantages. Specifically, the power consumption and losses increase with increased mode number while the bulk fluid flow decreases. Because power consumption must often meet very strict limitations in portable electronic devices, it is concluded that the fans used in this work should only be implemented at their first resonance mode for portable cooling applications.

These conclusions are valid for the length scale of commercially available piezoelectric fans. As the length scale is decreased, the surface area to volume ratio of the piezo element increases significantly, thereby effectively minimizing the dielectric losses. Moreover, the mechanical coupling factors $\left(\mathrm{Q}_{\mathrm{m}}\right)$ of microfabricated single crystal structures are usually high, suggesting that microfabricated piezoelectric fans operating in higher modes may realize the theoretical benefits expected of higher-mode operation, unlike the macrofans in the present work.

\section{REFERENCES}

[1] T. Acikalin, S. M. Wait, S. V. Garimella, and A. Raman, "Experimental investigation of the thermal performance of piezoelectric fans," Heat Transfer Eng. vol. 25, no. 1, pp. 4-14, 2004.

[2] S. M. Wait, T. Acikalin, S. V. Garimella, and A. Raman, "Piezoelectric fans for the thermal management of electronics," in Proc. Sixth ISHMT/ASME Heat and Mass Transfer Conf., Kalpakkam, India, January 5-7 2004, pp. 447-452.

[3] M. Toda, "Theory of air flow generation by a resonant type PVF2 bimorph cantilever vibrator," Ferroelectrics vol. 22, pp. 911-918, 1979.

[4] A. Ihara, and H. Watanabe, "On the flow around flexible plates oscillating with large amplitude," J. Fluid Struct. 8, 601-619 (1994).

[5] W. L. Nyborg, "Acoustic streaming near a boundary," J. Acoust. Soc. Am., vol. 30, pp. 329-339, 1958

[6] Y. H. Kim, S.T. Wereley, and C. H. Chun, "Phase-resolved flow field produced by a vibrating cantilever plate between two endplates," Phys. Fluids, vol. 16, no. 1, pp. 145-162, 2004.
[7] T. Acikalin, A. Raman , and S. V. Garimella, "Two-dimensional streaming flows induced by resonating thin beams," J. Acoust. Soc. Am, vol. 114, no. 4, pp. 1785-1795, 2003.

[8] N. T. Nguyen, and R. M. White, "Acoustic streaming in micromachined flexural plate wave devices: numerical simulation and experimental verification," 2000 IEEE T. Ultrason. Ferr., vol. 47 no. 6, pp. 14631471.

[9] R. R. Schmidt, "Local and average transfer coefficients on a vertical surface due to convection from a piezoelectric fan," in Proc. InterSoc. Conf. Therm. Phenom., pp. 41- 49, 1994.

[10] J. H. Yoo, J. I. Hong, and W. Cao, "Piezoelectric ceramic bimorph coupled to thin metal plate as cooling fan for electronic devices," Sensor Actuat. A-Phys., vol. 79, pp. 8-12, 2000.

[11] T. Wu, P. I. Ro, A. I. Kingon, and J. F. Mulling, 2003, "Piezoelectric resonating structures for microelectronic cooling," Smart Mater. Struct., vol.12, pp. 181-187, 2003.

[12] Q. Wan, and A. V. Kuznetsov, "Streaming in a channel bounded by an ultrasonically oscillating beam and its cooling efficiency," Numer. Heat Tr. A-Appl., vol. 45, pp. 21-47, 2004.

[13] B. G. Loh, S. Hyun, P. I. Ro, and C. Kleinstreuer, "Acoustic streaming induced by ultrasonic flexural vibrations and associated enhancement of convective heat transfer," J. Acous. Soc. Am., vol. 111, pp. 875-883, 2002.

[14] Y. S. Cho, Y. E. Pak, C. S. Han, and S. K. Ha, "Five-port equivalent circuit of piezoelectric bimorph beam," Sensor Actuat. A-Phys., vol 84, pp 140-148, 2000.

[15] D. A. Berlincourt, D. R. Curran, and H. Jaffe, "Piezoelectric and piezomagnetic materials and their function in transducers," Phys. Acoustics, vol. 1(A), pp. 170-267, 1964

[16] S. Basak, A. Raman, and S. V. Garimella, "Dynamic response optimization of piezoelectrically excited thin resonant beams," $J$ Vib. Acoust., vol. 127, pp. 18-27, 2005.

[17] IRE Standards on Piezoelectric Crystals, IRE Standard 49, pp. 1162$1169,1961$.

[18] ANSYS 8.0 Documentation, ANSYS Inc., 2004.

[19] Available at http://www.matweb.com/search/SearchSubcat.asp

[20] Available at http://www.piezo.com/

[21] T. Jordan, Z. Ounaies, J. Tripp, and P. Tcheng, "Electrical properties and power considerations of a piezoelectric actuator," Institute for Computer Applications in Science and Engineering, Hampton, VA ICASE 2000-8, 2000.

[22] K. Uchino, and S. Hirose, "Loss mechanisms in piezoelectrics: how to measure different losses separately," 2001 IEEE T. Ultrason. Ferr. vol. 48 , no. 1 , pp. 307-321. 
TABLE I. Piezoelectric fan parameters for Fan 1, Fan 2 and Fan 3. All the fans are constructed of a mylar shimstock with a Navy Type II PZT piezoceramic patch affixed on one side.

\begin{tabular}{llll}
\hline \hline & Fan 1 & Fan 2 & Fan 3 \\
PZT thickness $(\mathrm{cm})$ & 0.05 & 0.05 & 0.05 \\
Shim thickness $(\mathrm{cm})$ & 0.025 & 0.025 & 0.025 \\
L1 $(\mathrm{cm})$ & 0.3 & 0.3 & 0.3 \\
L2 $(\mathrm{cm})$ & 3.2 & 3.2 & 3.2 \\
L3 $(\mathrm{cm})$ & 6.85 & 6.15 & 4.19 \\
Fan width $(\mathrm{cm})$ & 1.3 & 1.3 & 1.3 \\
\hline \hline
\end{tabular}

TABLE II. Comparison of natural frequencies and EMCF values for Fan 1, Fan 2, and Fan 3 based on experimental measurements, finite element predictions, and observation of maximum flow.

\begin{tabular}{|c|c|c|c|c|c|c|c|c|c|c|}
\hline & & \multicolumn{3}{|c|}{ Impedance Analyzer } & \multicolumn{3}{|c|}{ ANSYS } & \multicolumn{3}{|c|}{ Observed (Visualization) } \\
\hline & & $\mathrm{f}_{\mathrm{m}}(\mathrm{Hz})$ & $\mathrm{f}_{\mathrm{n}}(\mathrm{Hz})$ & $\mathrm{EMCF}$ & $\mathrm{f}_{\mathrm{sc}}(\mathrm{Hz})$ & $\mathrm{f}_{\mathrm{oc}}(\mathrm{Hz})$ & EMCF & $\mathrm{f}_{\mathrm{res}}(\mathrm{Hz})$ & Power (mW) & $\tan \alpha$ \\
\hline \multirow[t]{4}{*}{ Fan 1} & $1^{\text {st }}$ & 59.2 & 59.9 & 0.023 & 58.351 & 58.354 & 0.011 & 60 & 1.99 & 0.31 \\
\hline & $2^{\text {nd }}$ & 238.5 & 251 & 0.097 & 236.218 & 236.337 & 0.032 & 265 & 16.15 & 0.77 \\
\hline & $3^{\mathrm{rd}}$ & 415 & 422 & 0.033 & 409.350 & 409.363 & 0.008 & 423 & 26.66 & 0.37 \\
\hline & $4^{\text {th }}$ & 1054 & 1060 & 0.011 & 1052.716 & 1052.717 & 0.002 & 1082 & 68.46 & 0.85 \\
\hline \multirow[t]{4}{*}{ Fan 2} & $1^{\text {st }}$ & 90.1 & 92 & 0.041 & 87.89 & 87.90 & 0.013 & 90.3 & 4.8 & 0.39 \\
\hline & $2^{\text {nd }}$ & 251 & 264.8 & 0.102 & 253.53 & 253.66 & 0.032 & 252.5 & 30.4 & 0.78 \\
\hline & $3^{\mathrm{rd}}$ & 620 & 621 & 0.003 & 599.50 & 599.51 & 0.002 & - & - & 0.52 \\
\hline & $4^{\text {th }}$ & 1565 & 1635 & 0.084 & 1464.26 & 1464.27 & 0.004 & 1582 & 83.7 & 1.32 \\
\hline \multirow[t]{4}{*}{ Fan 3} & $1^{\mathrm{st}}$ & 274 & 283.5 & 0.066 & 243.430 & 243.574 & 0.035 & 269 & 3.56 & 0.20 \\
\hline & $2^{\text {nd }}$ & - & - & - & 839.5286 & 839.5295 & 0.002 & - & - & - \\
\hline & $3^{\mathrm{rd}}$ & - & - & - & 1670.019 & 1670.029 & 0.003 & - & - & - \\
\hline & $4^{\text {th }}$ & - & - & - & 5770.930 & 5770.979 & 0.004 & - & - & - \\
\hline
\end{tabular}




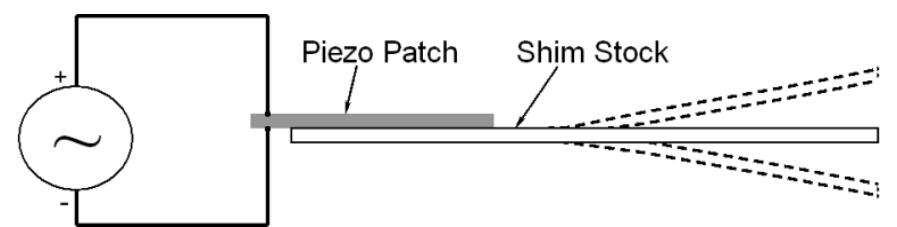

Fig. 1. Schematic of a piezoelectric fan oscillating under an applied voltage due to the contraction and expansion of a piezoceramic patch.

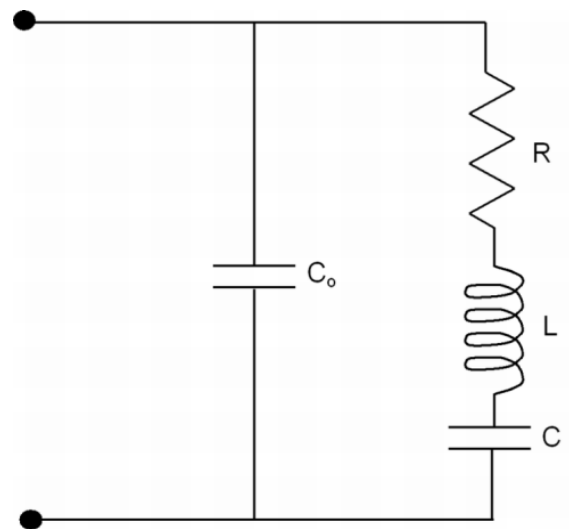

Fig. 2. Equivalent circuit diagram for a piezoelectric fan operating in a given resonance mode.

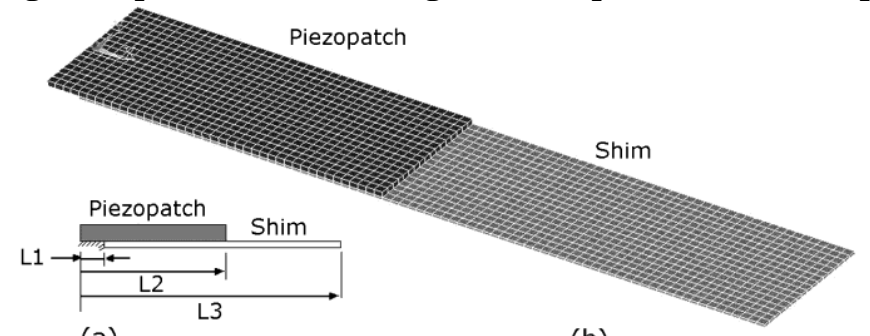

(a)

(b)

Fig. 3. Finite element model of the piezoelectric fan used in ANSYS. A grid size of $0.8 \mathrm{~mm}$ is used to create the mesh. 


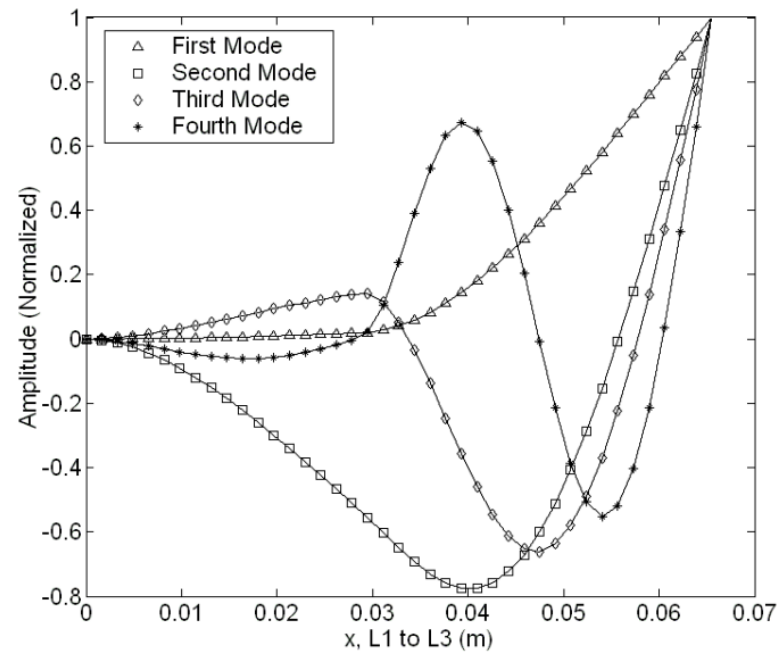

(a)

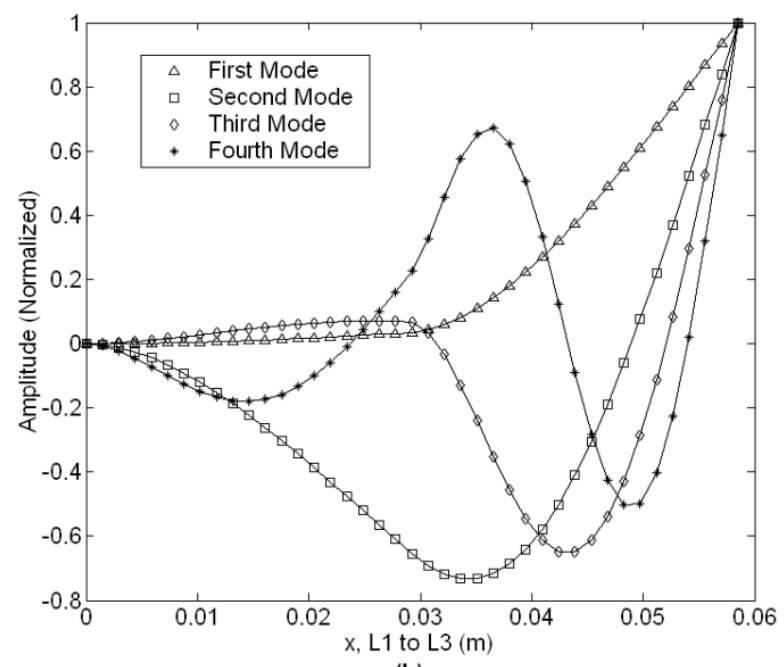

(b)

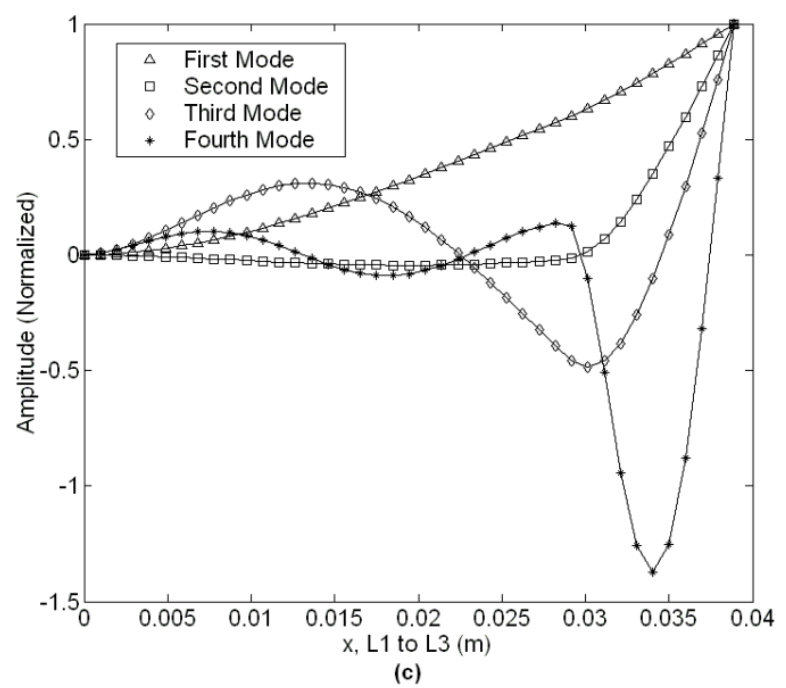

Fig. 4. Modal deflections for (a) Fan 1, (b) Fan 2, and (c) Fan 3 based on finite element analysis. 


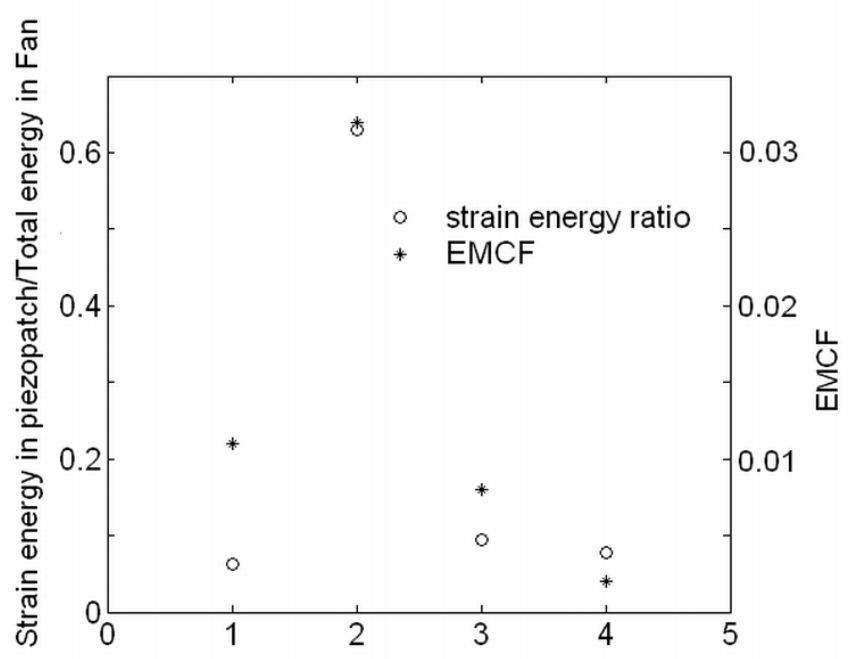

Fig. 5. The ratio of the strain energy in the piezopatch to the total strain energy in Fan 1, and the variation of the EMCF with increasing mode number are determined from finite element analysis. The two quantities compared show the same trend with mode number.

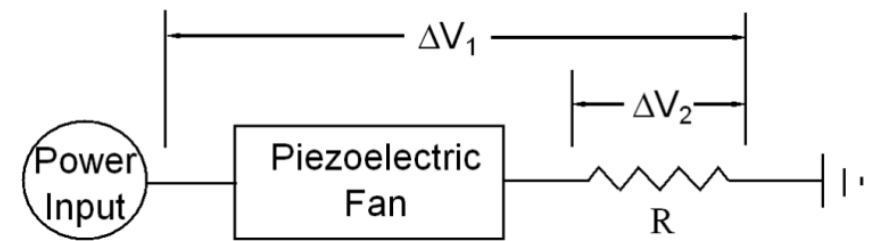

Fig. 6. Diagram for power consumption measurement: $\Delta V_{1}$ is measured between the power source and ground, and $\Delta V_{2}$ is measured across the resistor between the fan and ground.

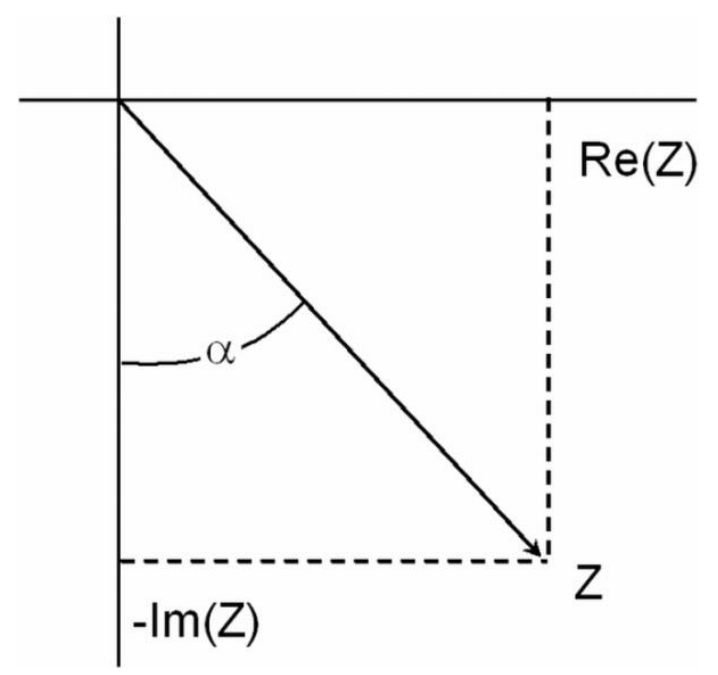

Fig. 7. Schematic illustration of impedance in the real-imaginary plane. 


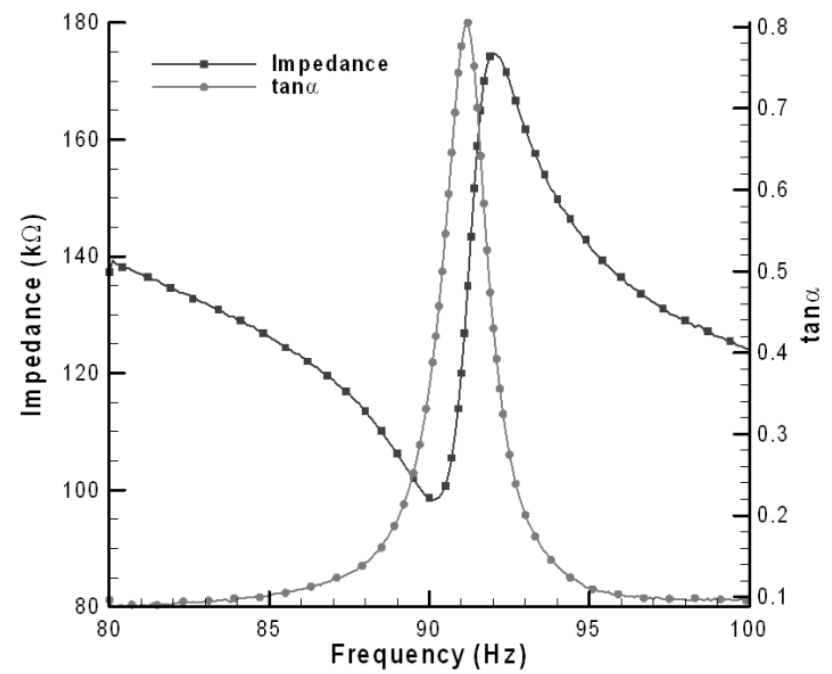

Fig. 8. Experimentally measured impedance and dissipation factor for Fan 2 near the fundamental resonance frequency of $90.3 \mathrm{~Hz}$. The loss factor is largest when operating off resonance. 


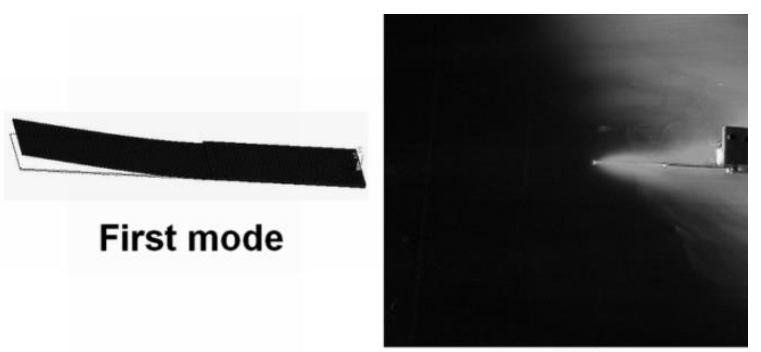

$t=1 / 15 s$

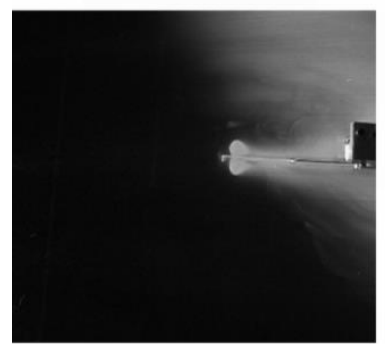

$t=2 / 15 s$

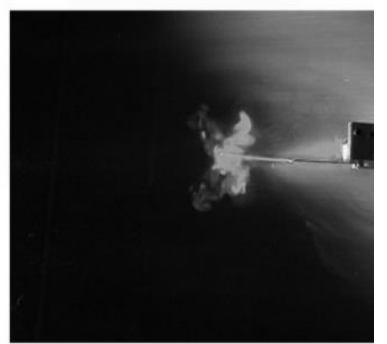

$t=4 / 15 s$
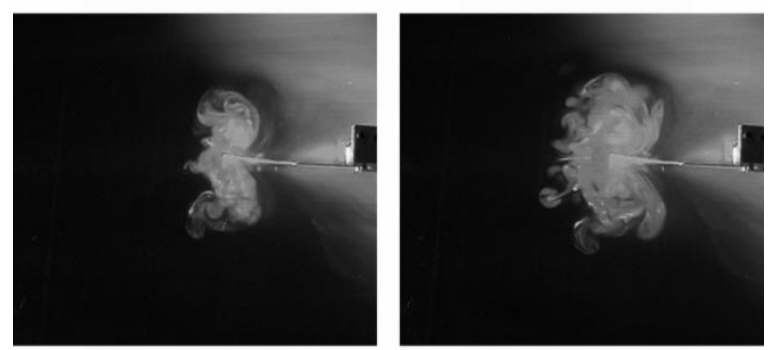

$t=6 / 15 s$

$t=8 / 15 s$

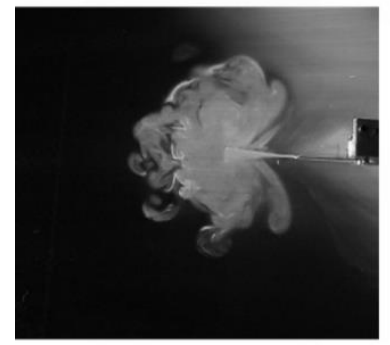

$t=10 / 15 s$

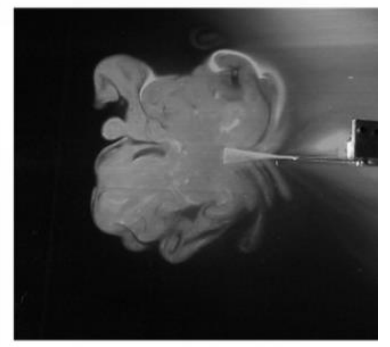

$t=15 / 15 s$

Fig. 9. Flow visualization for Fan 1 operating at the fundamental resonance frequency of $60 \mathrm{~Hz}$. 


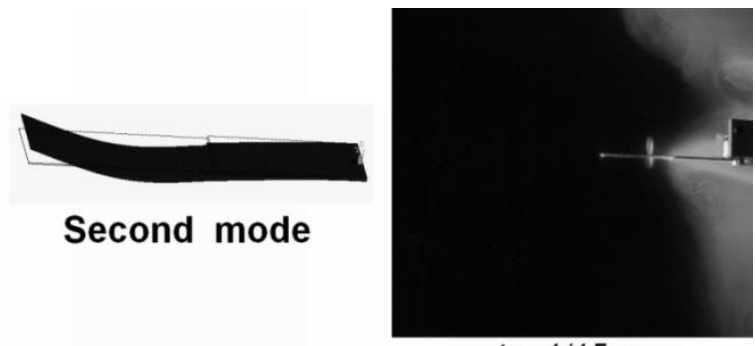

$t=1 / 15 s$

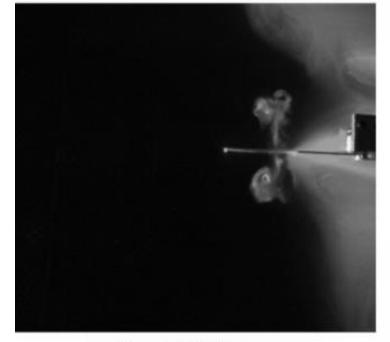

$t=2 / 15 s$

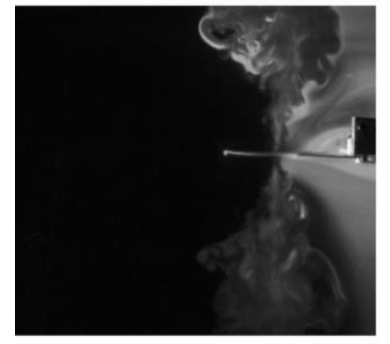

$t=6 / 15 s$

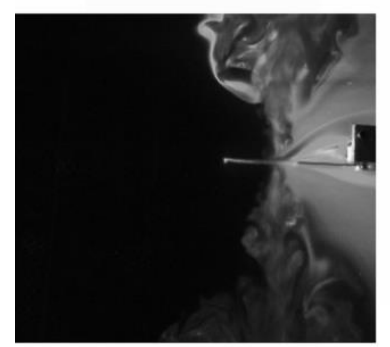

$t=10 / 15 s$

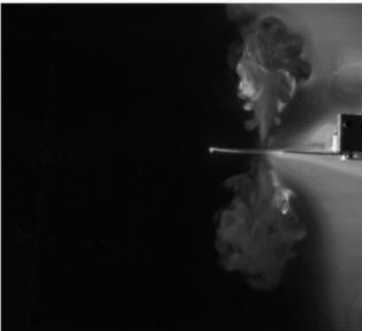

$\mathrm{t}=4 / 15 \mathrm{~s}$

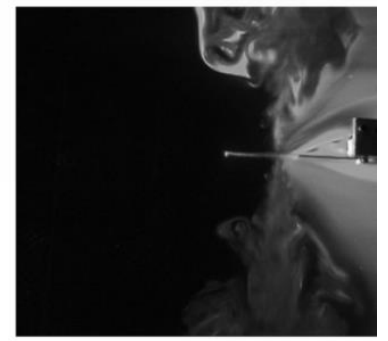

$t=8 / 15 s$

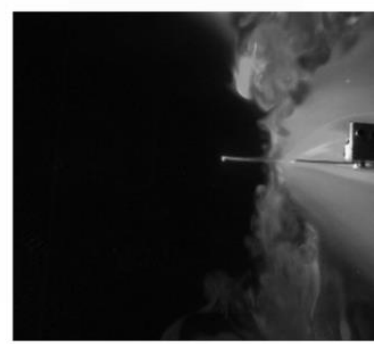

$t=15 / 15 s$

Fig. 10. Flow visualization for Fan 1 operating at the second resonance frequency of $265 \mathrm{~Hz}$. 


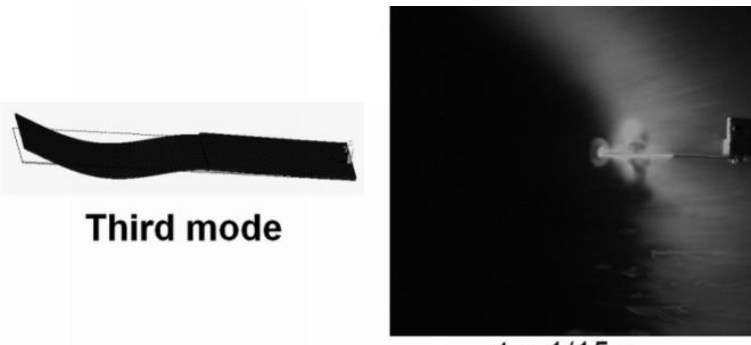

$t=1 / 15 s$

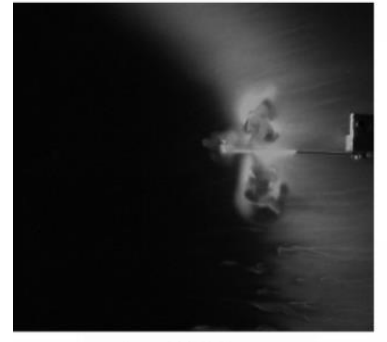

$t=2 / 15 s$

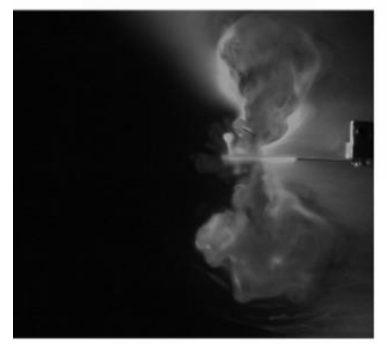

$t=6 / 15 s$

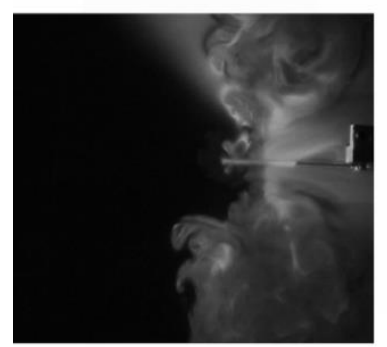

$t=10 / 15 s$

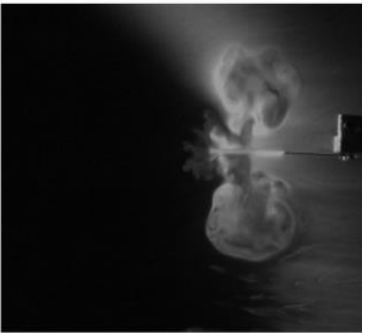

$t=4 / 15 s$

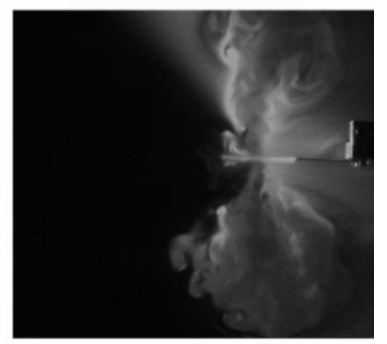

$t=8 / 15 s$

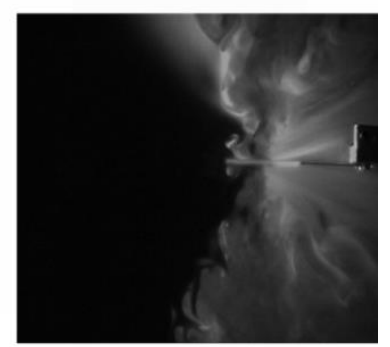

$t=15 / 15 s$

Fig. 11. Flow visualization for Fan 1 operating at the third resonance frequency of $423 \mathrm{~Hz}$. 


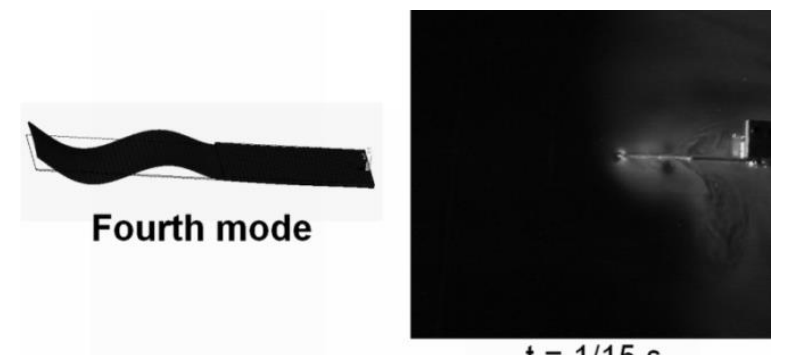

$t=1 / 15 s$

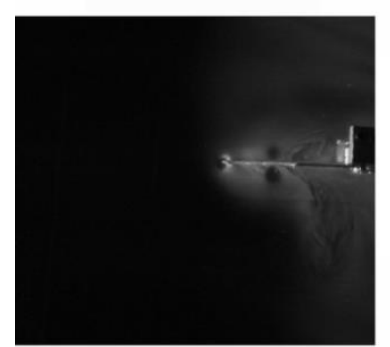

$t=2 / 15 s$

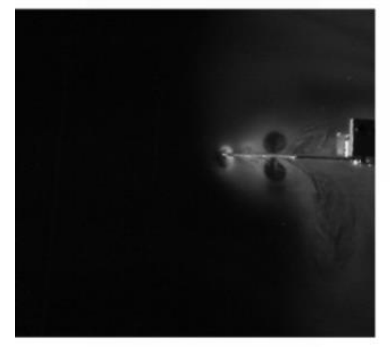

$t=6 / 15 s$

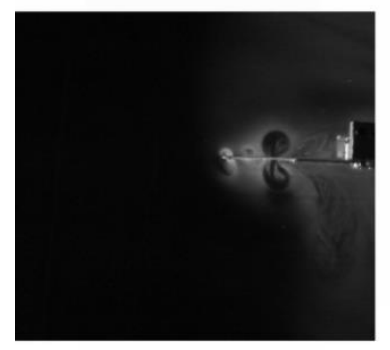

$t=10 / 15 s$

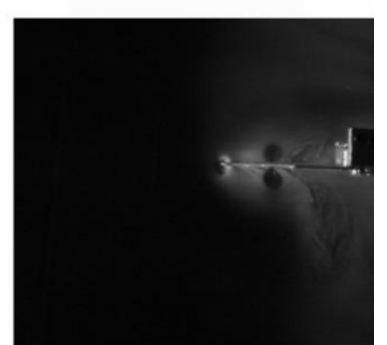

$t=4 / 15 \mathrm{~s}$

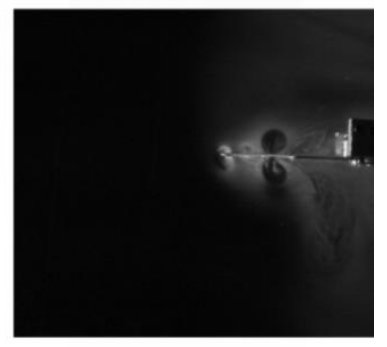

$\mathrm{t}=8 / 15 \mathrm{~s}$

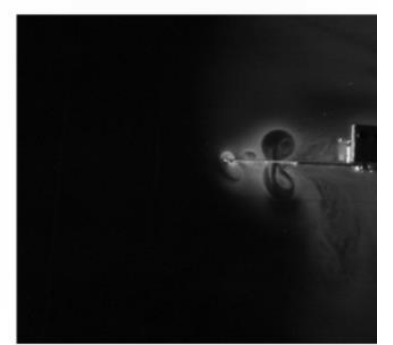

$t=15 / 15 s$

Fig. 12. Flow visualization for Fan 1 operating at the fourth resonance frequency of $1082 \mathrm{~Hz}$. 


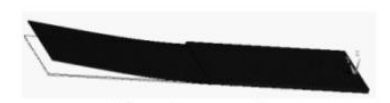

First mode

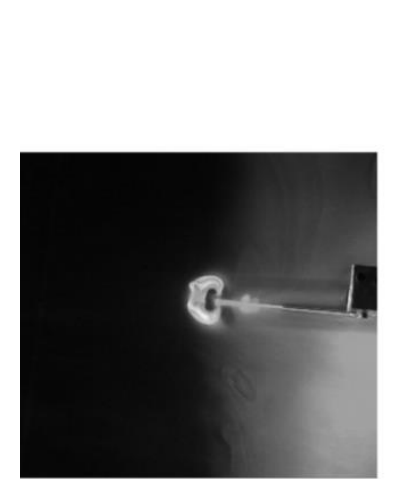

$t=2 / 15 s$

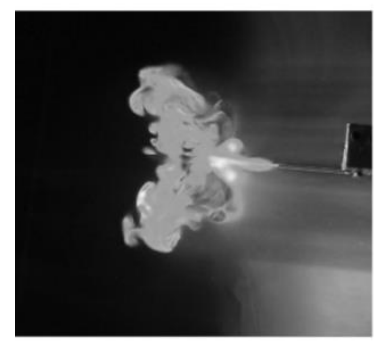

$t=6 / 15 s$

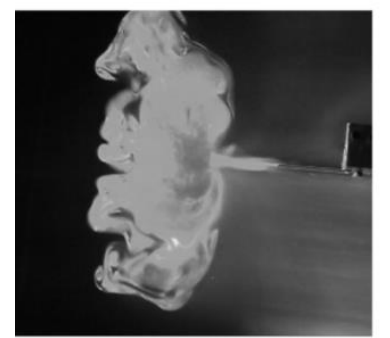

$t=10 / 15 s$

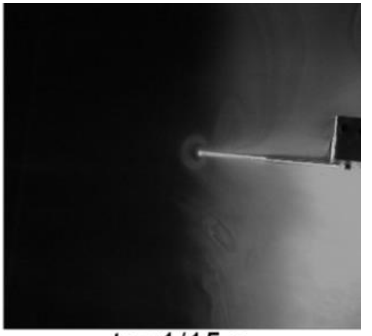

$t=1 / 15 s$

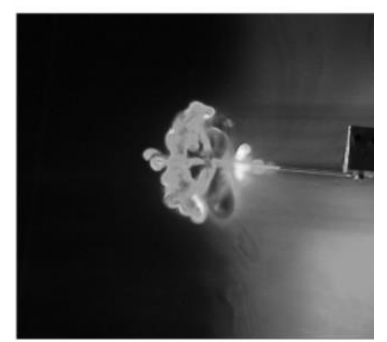

$t=4 / 15 s$

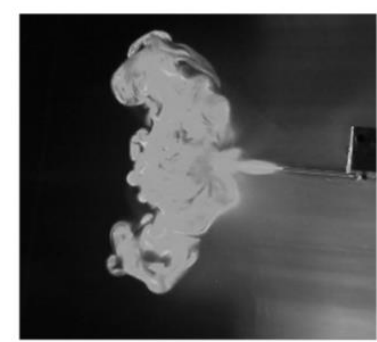

$t=8 / 15 \mathrm{~s}$

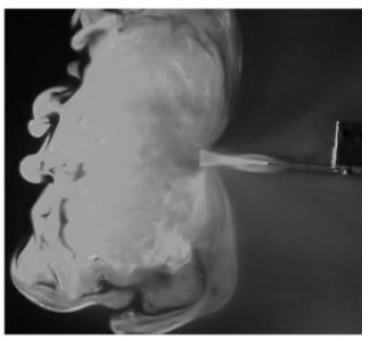

$t=15 / 15 \mathrm{~s}$

Fig. 13. Flow visualization for Fan 2 operating at the fundamental resonance frequency of $90.3 \mathrm{~Hz}$. 


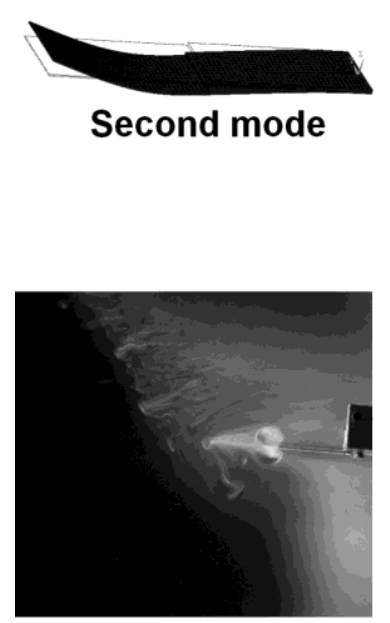

$t=2 / 15 s$

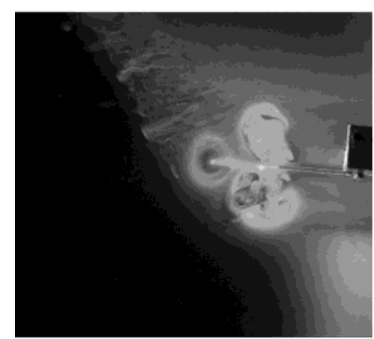

$t=6 / 15 s$

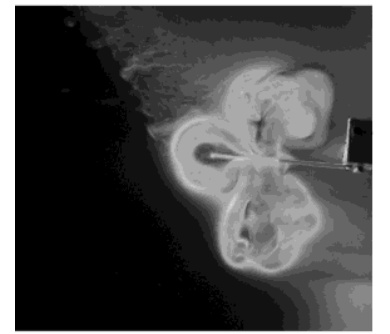

$t=10 / 15 s$

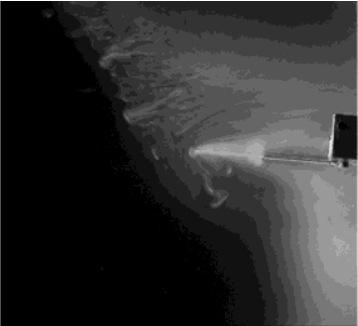

$t=1 / 15 s$

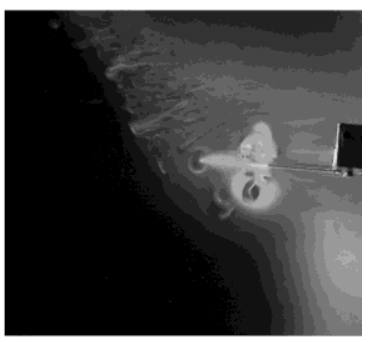

$t=4 / 15 s$

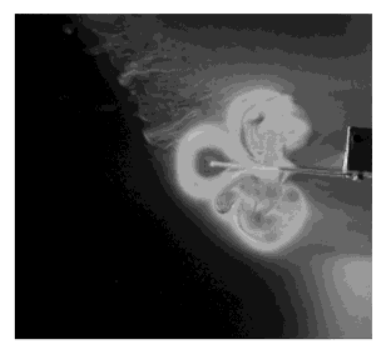

$\mathrm{t}=8 / 15 \mathrm{~s}$

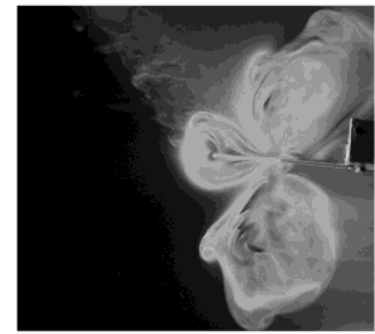

$t=15 / 15 s$

Fig. 14. Flow visualization for Fan 2 operating at the second resonance frequency of $252.5 \mathrm{~Hz}$. 


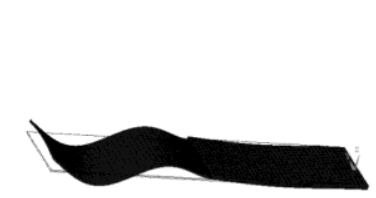

Fourth mode

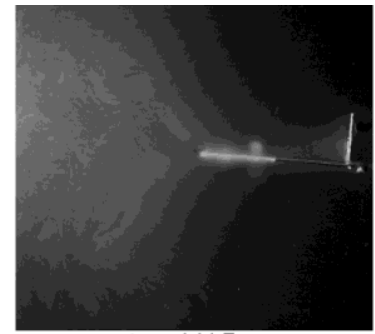

$t=1 / 15 s$
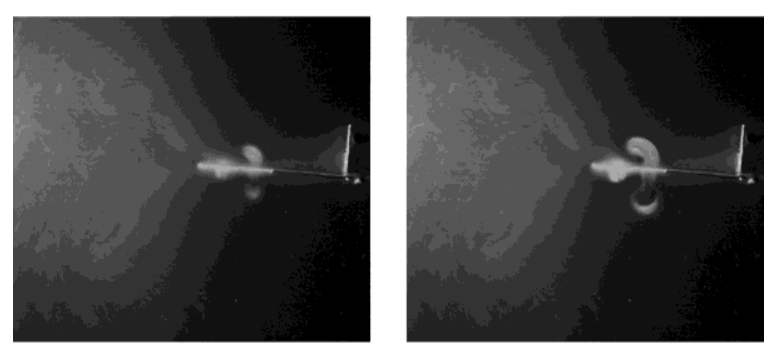

$t=2 / 15 s$

$t=4 / 15 s$

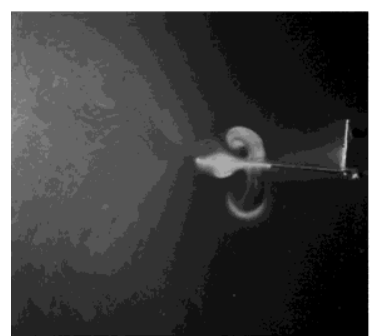

$t=6 / 15 s$

$t=8 / 15 s$

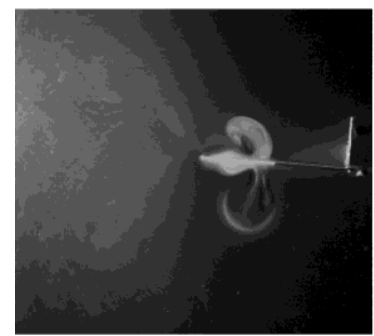

$t=10 / 15 s$
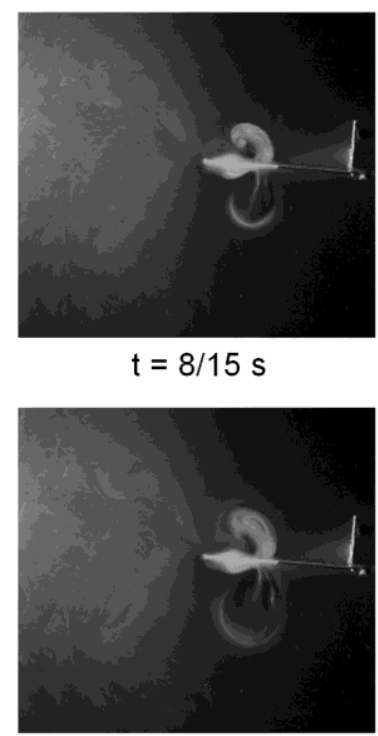

$t=15 / 15 s$

Fig. 15. Flow visualization for Fan 2 operating at the fourth resonance frequency of $1582 \mathrm{~Hz}$. 


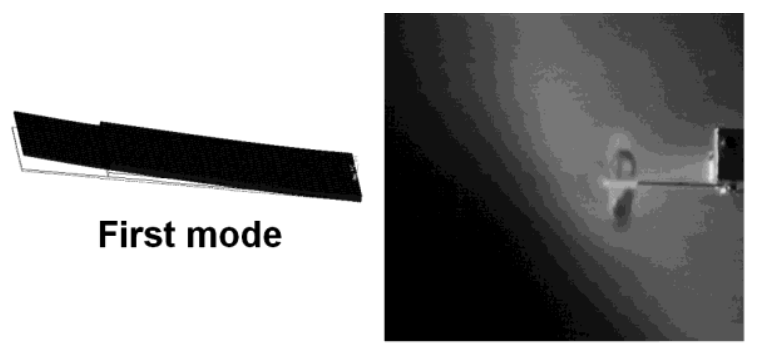

$t=1 / 15 s$

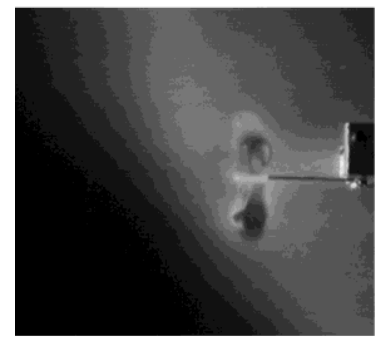

$t=2 / 15 s$

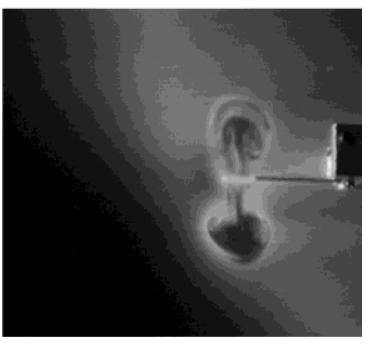

$t=4 / 15 s$

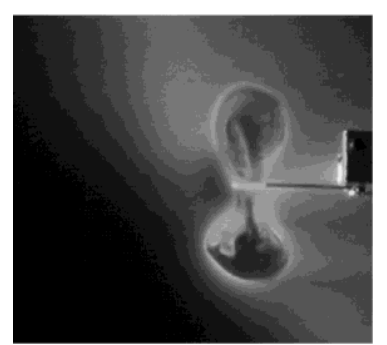

$t=6 / 15 s$

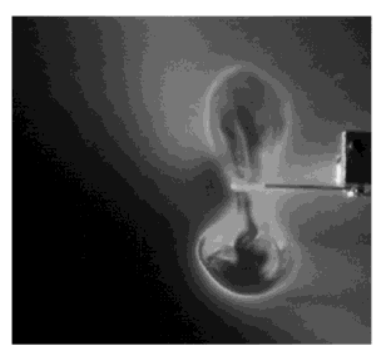

$t=8 / 15 s$

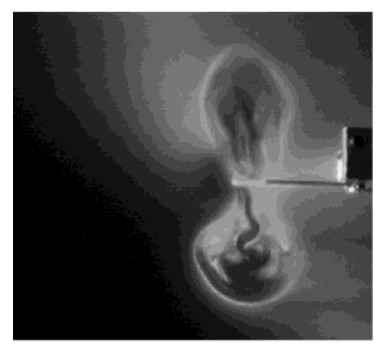

$t=10 / 15 s$

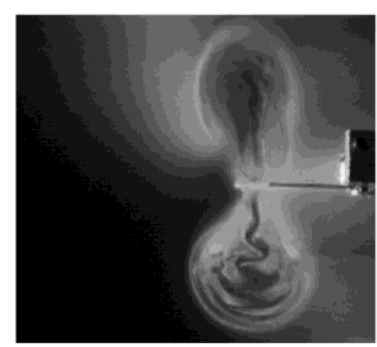

$t=15 / 15 s$

Fig. 16. Flow visualization for Fan 3 operating at the fundamental resonance frequency of $269 \mathrm{~Hz}$. 